\title{
The Adherence Rate Threshold is Drug Specific
}

\author{
Melissa E. Stauffer $^{1} \cdot$ Paul Hutson $^{2} \cdot$ Anna S. Kaufman $^{1} \cdot$ Alan Morrison $^{1}$
}

Published online: 26 October 2017

(C) The Author(s) 2017. This article is an open access publication

\begin{abstract}
Introduction Patient adherence to a medication regimen is usually expressed as an adherence rate, defined as the proportion of prescribed doses actually taken. An adherence rate threshold, above which the therapeutic effect is maintained, is typically assigned an arbitrary value, commonly 0.8 .

Objective Here, we determined the value of the adherence rate threshold objectively in different drugs of the same class, using statins as an example.

Methods We used pharmacokinetic/pharmacodynamic (PK/PD) modeling to predict serum levels of low-density lipoprotein cholesterol (LDL-C) in patients taking simvastatin $20 \mathrm{mg}$ or atorvastatin $5 \mathrm{mg}$ once daily for 30 days. LDL-C reduction was modeled for adherence rates of 1.0, $0.8,0.6,0.4$, and 0.2. The results were expressed as the percentage of time spent at the LDL-C goal $(<70 \mathrm{mg} / \mathrm{dL})$. The adherence rate threshold was defined as the minimum adherence rate that resulted in the same amount of time at goal as perfect adherence (i.e., a rate of 1.0).

Results For simvastatin, an adherence rate of 0.8 resulted in a significant decrease in time at the LDL-C goal compared to perfect adherence $(54.8 \%$ versus $85.1 \%$; $P<0.001$ ), and rates $<0.8$ resulted in progressively less time at goal. For atorvastatin, the rates of 0.8 and 0.6 resulted in essentially the same amount of time at goal as perfect adherence $(87.8 \%$ and $87.7 \%$, respectively, versus $88.1 \% ; P>0.05$ for both), with less time at goal only
\end{abstract}

Melissa E. Stauffer

melstauff@charter.net

ScribCo, Effort, PA, USA

2 University of Wisconsin-Madison School of Pharmacy, Madison, WI, USA occurring at rates $\leq 0.4(P<0.001)$. Thus, the adherence rate thresholds are $>0.8$ for simvastatin and between 0.4 and 0.6 for atorvastatin.

Conclusion These results indicate that a value of 0.8 cannot be applied universally.

\section{Key Points}

We performed pharmacokinetic/pharmacodynamic (PK/PD) simulations of the effects on serum lowdensity lipoprotein cholesterol (LDL-C) levels of various rates of adherence to simvastatin $20 \mathrm{mg}$ and atorvastatin $5 \mathrm{mg}$.

The adherence rate threshold, above which serum levels of LDL-C were maintained in the target range, was $>0.8$ for simvastatin and between 0.4 and 0.6 for atorvastatin.

We conclude that there is no unique adherence rate threshold, e.g., 0.8, that is universally applicable to all drugs.

\section{Introduction}

In retrospective studies, medication adherence is often quantified as the medication possession ratio (MPR), that is, the number of dispensed doses of a drug divided by the number of doses prescribed in a specified time period $[1,2]$. An MPR of 0.8 is commonly considered an acceptable level of adherence [3]. However, this threshold is often applied with no clinical rationale [2], despite evidence that values other than 0.8 may provide therapeutic 
efficacy [4-7] and that, in some cases, there is no threshold, but rather a continuous relationship between adherence and efficacy [8].

We previously described how a threshold for adherence can be determined objectively, based on a drug's forgiveness, that is, the difference between the duration of its therapeutic effect and the dosing interval [9]. In this context, adherence is quantified as a rate and is defined as the proportion of prescribed doses consumed within a defined time period. The adherence rate threshold is the lowest rate at which the desired therapeutic effect is maintained.

The aim of the current study was to estimate the value of the adherence rate threshold using a different method: pharmacokinetic/pharmacodynamic (PK/PD) modeling. We determined whether the adherence rate threshold differs between drugs in the same class, using as a model system the 3-hydroxy-3-methyl-glutaryl-coenzyme A (HMG-CoA) reductases (i.e., statins), because of their diverse PK/PD properties.

\section{Methods}

\subsection{Study Design}

This was a PK/PD modeling study of the clinical effects of non-adherence based on known statin PK/PD parameters and medication event monitoring system (MEMS) data. We used PK/PD modeling to predict serum levels of lowdensity lipoprotein cholesterol (LDL-C) in patients taking simvastatin or atorvastatin once daily for 30 days. Variable adherence was incorporated into the model as adherence rates ranging from 0.2 to 1.0. Predicted serum concentration profiles of LDL-C were generated, and the percentage of time spent at the LDL-C goal was computed, allowing us to determine the adherence rate threshold for each statin.

\subsection{Dosing Regimens}

MEMS data were obtained from the iAdherence database (www.iadherence.org) [10]. This database contains MEMS records for 833 patients taking a once-daily drug for hypercholesterolemia for a duration of up to 300 days. Each patient has a numeric identifier-a (non-sequential) number between 4001 and 67,456. The data set records the date and time when each dose was taken, but does not include any patient-identifying information or the identity of the specific drug.

MEMS data for 30-day periods were extracted from the iAdherence database for a heuristic sample of five patients with dosing patterns corresponding to adherence rates of 1.0, 0.8, 0.6, 0.4, and 0.2 (Table 1). These dosing patterns consisted of $0,6,12,18$, and 24 missed doses, respectively
Table 1 Characteristics of the patient sample

\begin{tabular}{lll}
\hline Patient $^{I^{\mathrm{a}}}{ }^{\mathrm{a}}$ & Missed doses $^{\mathrm{b}}$ & Adherence rate \\
\hline 4002 & 0 & 1.0 \\
4016 & 6 & 0.8 \\
4051 & 12 & 0.6 \\
4084 & 18 & 0.4 \\
4287 & 24 & 0.2 \\
\hline
\end{tabular}

${ }^{\mathrm{a}}$ ID numbers are from iadherence.org

${ }^{\mathrm{b}}$ Number of misses in the 30-day window

(i.e., 30, 24, 18, 12, and 6 doses consumed, respectively; Fig. 1). The 30-day period selected was not constrained to any particular time during the treatment regimen (e.g., the first 30 or last 30 days of treatment). For each of the five patients, the time of each dose was recorded in Microsoft Excel. Dose times were rounded to the nearest hour ( $\geq 30 \mathrm{~min}$ past the hour was rounded up; $<30 \mathrm{~min}$ was rounded down) and then converted into a series of time points for use by the PK/PD modeling program.

\subsection{Pharmacokinetic/Pharmacodynamic Modeling}

PK/PD modeling was performed using the SIM module of ADAPT v. 5 [11]. We used doses of simvastatin $20 \mathrm{mg}$ and atorvastatin $5 \mathrm{mg}$, based on a systematic review of clinical trials showing that these doses would be expected to produce the same $\sim 30 \%$ reduction in LDL-C [12]. Parameters required for PK/PD modeling of simvastatin [13] and atorvastatin [14] were obtained from published studies. The pmlag model file from the ADAPT library was modified as needed to use the published PK data for each statin. The PK parameter names and values are shown in Tables 2 and 3, and schematic diagrams of the models are shown in Fig. 2.

Among the published PD indirect response models for statins [13, 15, 16], the model described in Kim et al. [13] was found to be the best match to the published PK/PD data for both simvastatin and atorvastatin. This model assumes inhibition of LDL-C production by a factor of $\left(1-\left(E_{\max } \times C_{\mathrm{t}}\right) /\left(\mathrm{EC}_{50}+C_{\mathrm{t}}\right)\right)$, where $E_{\max }$ is the maximum inhibitory effect (a unitless number between 0 and 1), $\mathrm{EC}_{50}$ is the statin concentration producing half-maximal inhibition, and $C_{\mathrm{t}}$ is the concentration of the active metabolite of the statin. To ensure the internal consistency of the model for both statins, the $\mathrm{EC}_{50}$ values were obtained from a study of HMG-CoA reductase activity in human liver microsomes [17].

\subsection{Clinical Scenario}

The relationship between the adherence rate and the therapeutic effect was assessed within a cardiovascular 
Fig. 1 Dosing patterns for each adherence rate. Shown are the dosing patterns for the five patients listed in Table 1. Each bar represents a dose taken, and misses are indicated by missing bars

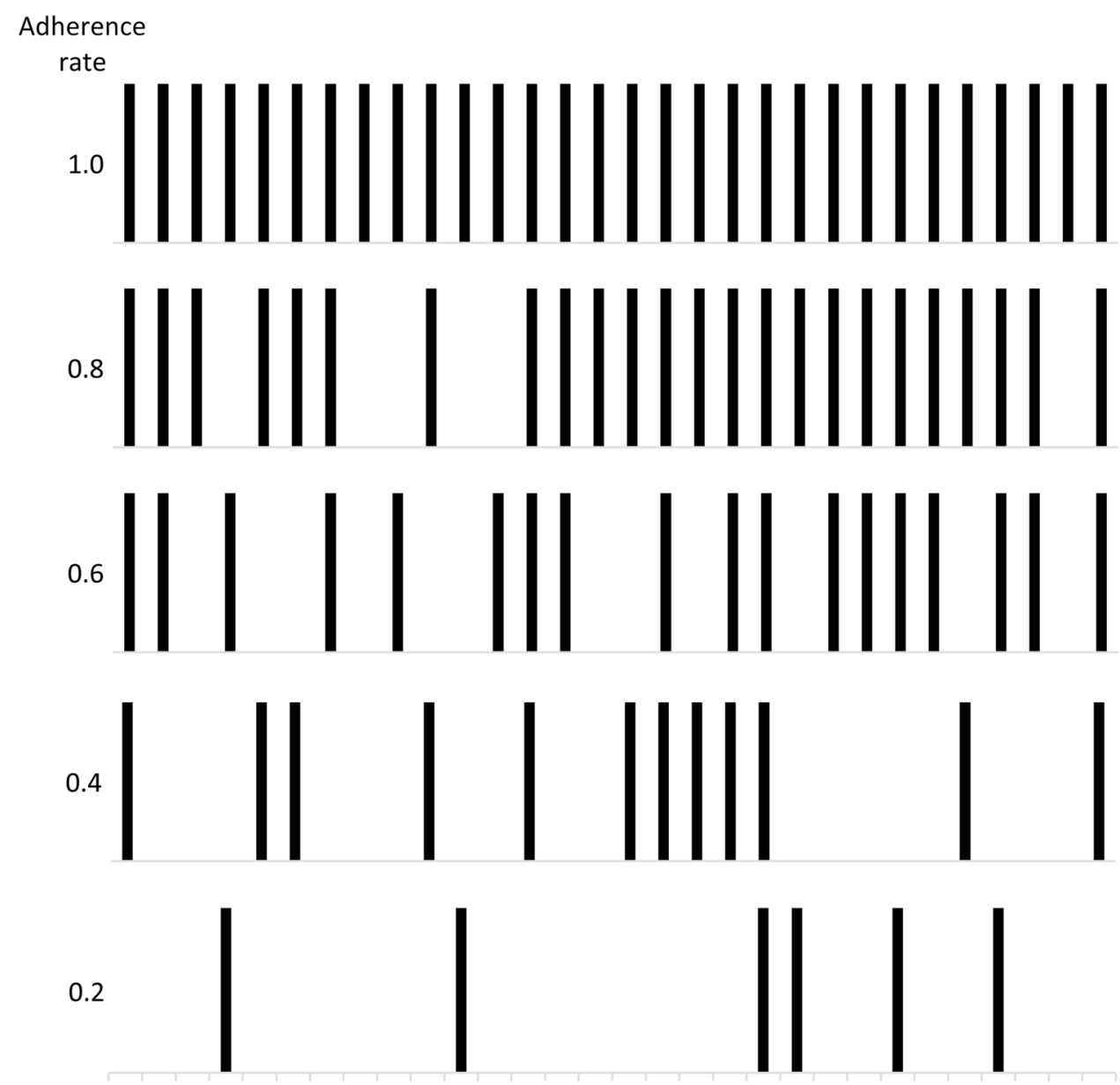

$122 \quad 3 \quad 4 \quad 5 \quad 6 \quad 7 \quad 8 \quad 9101112131415161718192021222324252627282930$ prevention scenario in which a patient with an initial LDLC level of $100 \mathrm{mg} / \mathrm{dL}$ took simvastatin or atorvastatin to achieve an LDL-C goal of $<70 \mathrm{mg} / \mathrm{dL}$. This LDL-C goal corresponds to both the target level recommended by the 2016 Guidelines of the European Society for Cardiology and the European Atherosclerosis Society for very high risk patients [18] and to the $\geq 30 \%$ reduction recommended by the American College of Cardiology/American Heart Association for patients expected to benefit from treatment with a moderate-intensity statin [19].

\subsection{Statistical Analysis}

The PK/PD simulations produced LDL-C concentration profiles consisting of 1000 data points over 30 days $(720 \mathrm{~h})$. The increment of time associated with each data point (approximately $0.7 \mathrm{~h}$ ) was calculated, and a binary (yes/no) determination was made of whether that increment was spent at the LDL-C goal $(<70 \mathrm{mg} / \mathrm{dL})$. Summing the 'yes' increments and dividing by the total time course gave the percentage of time for which the serum LDL-C level was at goal. The adherence rate threshold was defined as the lowest adherence rate that facilitated attainment of the LDL-C goal for the same amount of time as perfect adherence (i.e., 1.0), subject to a $z$ test of proportions. $Z$ tests were performed in SigmaPlot v. 12 .

\section{Results}

\subsection{Low-Density Lipoprotein Cholesterol (LDL-C) Reduction at Various Adherence Rates}

The predicted levels of LDL-C in patients taking simvastatin $20 \mathrm{mg}$ or atorvastatin $5 \mathrm{mg}$ are shown in Fig. 3. With perfect adherence (a rate of 1.0), LDL-C levels decreased to $\sim 60 \mathrm{mg} / \mathrm{dL}$ with simvastatin $20 \mathrm{mg}$ and to $\sim 50 \mathrm{mg} /$ $\mathrm{dL}$ with atorvastatin $5 \mathrm{mg}$. The simvastatin curves showed sensitivity to the adherence rate, i.e., each curve was different from the others, across the entire range of rates, whereas the atorvastatin curves were very similar for 
Table 2 PK/PD model parameters for simvastatin

Table 3 PK/PD model parameters for atorvastatin

\begin{tabular}{lll}
\hline ADAPT parameter & Value & Corresponding parameter in Kim et al. [13] \\
\hline PK component & & \\
$K_{21}\left(\mathrm{~h}^{-1}\right)$ & 2.76 & $K_{\mathrm{a}}$ \\
$V_{1}(\mathrm{~L})$ & 8980 & $V_{2}$ \\
$K_{10}\left(\mathrm{~h}^{-1}\right)^{\mathrm{a}}$ & 0.136 & Calculated from $\mathrm{CL}_{2} / V_{2}$ (see below) \\
$K_{14}\left(\mathrm{~h}^{-1}\right)^{\mathrm{a}}$ & 0.058 & Calculated from $\mathrm{CL}_{2} / V_{2}$ (see below) \\
$V_{4}(\mathrm{~L})$ & 1190 & $V_{3}$ \\
$K_{40}\left(\mathrm{~h}^{-1}\right)^{\mathrm{b}}$ & 0.322 & Calculated from $\mathrm{CL}_{3} / V_{3}$ (see below) \\
Tau $(\mathrm{h})$ & 0.212 & Lag \\
$\mathrm{PD} \mathrm{component}$ & & \\
$k_{\text {in }}(\mathrm{mg} / \mathrm{dL} \cdot \mathrm{h})$ & 1.14 & $k_{\text {in }}$ \\
$E_{\text {max }}$ & 0.489 & $E_{\text {max }}$ \\
$\mathrm{EC}$ & 66.97 & $-{ }^{\mathrm{c}}$ \\
$k_{\text {out }}(\mathrm{mg} / \mathrm{mL} / \mathrm{dL} \cdot \mathrm{h}) /(\mathrm{mg} / \mathrm{dL})$ & 0.0114 & $k_{\text {in }} / \mathrm{baseline} \mathrm{LDL-C}$ \\
\hline
\end{tabular}

The following model parameters were set to zero: $V_{3}, K_{13}, K_{31}, V_{5}, K_{45}$, and $K_{54}$

For the ADAPT parameters, single-digit subscripts correspond to the compartment numbers in the models. Two-digit subscripts indicate flux between numbered compartments

$C L$ clearance, $E C_{50}$ concentration producing half-maximal inhibition, $E_{\max }$ maximum inhibitory effect, $K \mathrm{CL} / \mathrm{V}, m$ metabolite, $p$ parent compound, $P D$ pharmacodynamic, $P K$ pharmacokinetic, $Q$ intercompartmental clearance, $V$ volume

${ }^{\mathrm{a} C a l c u l a t e d}$ from $\mathrm{CL}_{2}$ and $V_{2}$ in Kim et al. [13]. $K_{10}=K_{1} \times 0.7, K_{14}=K_{1} \times 0.3 . K_{1}=\mathrm{CL}_{2} / V_{2}=1740 /$ $8980=0.194 \mathrm{~h}^{-1}$

${ }^{\mathrm{b}}$ Calculated from $\mathrm{CL}_{3}$ and $V_{3}$ in Kim et al. [13]. $K_{40}=\mathrm{CL}_{3} / V_{3}=383 / 1190=0.322 \mathrm{~h}^{-1}$

${ }^{\mathrm{c}} \mathrm{EC}_{50}$ values were obtained from Dansette et al. [17]

\begin{tabular}{lll}
\hline ADAPT parameter & Value & Corresponding parameter in Narwal et al. [14] \\
\hline PK component & & \\
$K_{21}\left(\mathrm{~h}^{-1}\right)$ & 3.5 & $K_{\mathrm{a}}$ \\
$V_{1}(\mathrm{~L})$ & 3250 & $V_{1}$ \\
$V_{3}(\mathrm{~L})^{\mathrm{a}}$ & 2170 & $V_{2}$ \\
$K_{13}\left(\mathrm{~h}^{-1}\right)$ & 0.578 & $Q / V_{1}$ \\
$K_{31}\left(\mathrm{~h}^{-1}\right)$ & 0.866 & $Q / V_{3}$ \\
$K_{14}\left(\mathrm{~h}^{-1}\right)$ & 0.155 & $\mathrm{CL}_{\mathrm{pm}} / V_{1}$ \\
$K_{41}\left(\mathrm{~h}^{-1}\right)$ & 0.175 & $\mathrm{CL}_{\mathrm{mp}} / V_{\mathrm{m}}$ \\
$V_{4}(\mathrm{~L})$ & 137 & $V_{\mathrm{m}}$ \\
$K_{40}\left(\mathrm{~h}^{-1}\right)$ & 0.847 & $\mathrm{CL}_{\mathrm{m}} / V_{\mathrm{m}}$ \\
$\mathrm{PD}$ component & & \\
$k_{\text {in }}(\mathrm{mg} / \mathrm{dL} \cdot \mathrm{h})$ & 1.14 & $-{ }^{\mathrm{b}}$ \\
$E_{\mathrm{max}}$ & 0.489 & $-{ }^{\mathrm{b}}$ \\
$\mathrm{EC}_{50}(\mathrm{ng} / \mathrm{mL})$ & 22.3 & ${ }^{\mathrm{c}}$ \\
$k_{\text {out }}(\mathrm{mg} / \mathrm{dL} \cdot \mathrm{h}) /(\mathrm{mg} / \mathrm{dL})$ & 0.0114 & $-{ }^{\mathrm{b}}$ \\
\hline
\end{tabular}

The following model parameters were set to zero: $K_{10}, V_{5}, K_{45}, K_{54}$, and $\tau$

For the ADAPT parameters, single-digit subscripts correspond to the compartment numbers in the models. Two-digit subscripts indicate flux between numbered compartments

$C L$ clearance, $E C_{50}$ concentration producing half-maximal inhibition, $E_{\max }$ maximum inhibitory effect, $K \mathrm{CL} / \mathrm{V}, m$ metabolite, $p$ parent compound, $P D$ pharmacodynamic, $P K$ pharmacokinetic, $Q$ intercompartmental clearance, $V$ volume

${ }^{a}$ Calculated as a secondary parameter in ADAPT

${ }^{\mathrm{b}}$ From Kim et al. [13]

${ }^{\mathrm{c}}$ From Dansette et al. [17] 
Fig. 2 Compartmental PK/PD models for a simvastatin and b atorvastatin. Dashed lines indicate inhibition of LDL-C production. $L D L-C$ low-density lipoprotein cholesterol, $P D$ pharmacodynamic, $P K$ pharmacokinetic
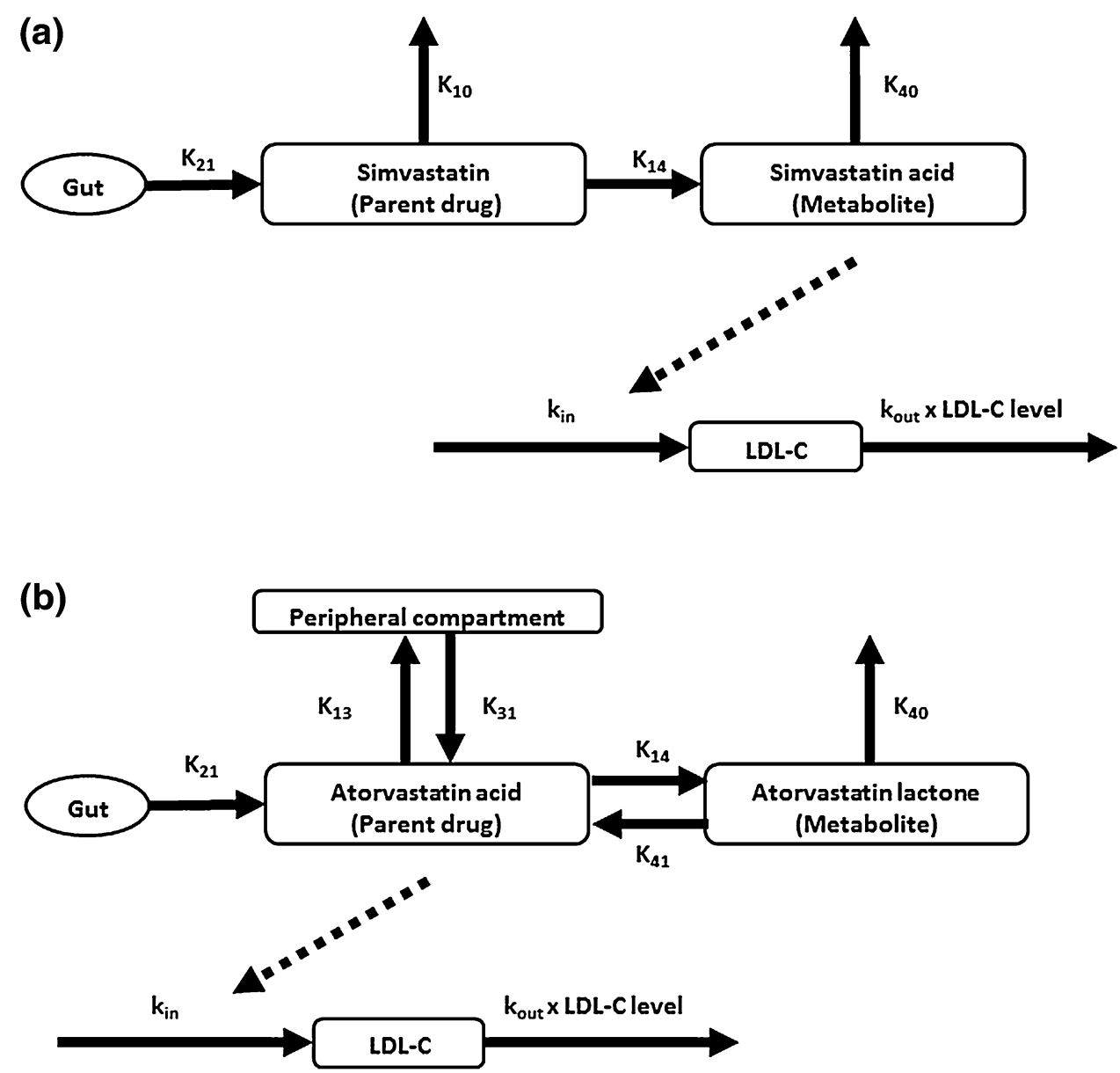

adherence rates of $1.0,0.8$, and 0.6 , showing more variability only at rates of 0.4 and 0.2 .

\subsection{Time at the LDL-C Goal}

The percentage of time spent at the LDL-C goal of $<70 \mathrm{mg} / \mathrm{dL}$ is shown in Fig. 4. For simvastatin $20 \mathrm{mg}$, an adherence rate of 0.8 resulted in a significant loss of time at the LDL-C goal compared to perfect adherence (54.8 vs $85.1 \% ; P<0.001)$, and rates $<0.8$ resulted in progressively less time at goal $(21.1,8.3$, and $0.0 \%$, for $0.6,0.4$, and 0.2 , respectively). For atorvastatin $5 \mathrm{mg}$, adherence rates of 0.8 and 0.6 allowed attainment of the LDL-C goal for essentially the same amount of time as perfect adherence $(87.8$ and $87.7 \%$, respectively, vs $88.1 \% ; P>0.05$ for both), with a reduction of time at goal only occurring at rates $\leq 0.4$ (63.5\% for 0.4 and $22.4 \%$ for $0.2 ; P<0.001$ for both). Based on the data in Fig. 4 and our definition of the adherence rate threshold, the threshold for simvastatin $20 \mathrm{mg}$ was $>0.8$ and that for atorvastatin $5 \mathrm{mg}$ was between 0.4 and 0.6 .

\section{Discussion}

Patient adherence patterns have previously been incorporated into $\mathrm{PK} / \mathrm{PD}$ modeling, with applications in clinical trial design and drug development $[20,21]$. A number of studies have quantified the relationship between adherence, drug exposure, and clinical effect using PK/PD models, producing metrics for "pharmacokinetic adherence" based on the mean and trough serum drug levels, the variance of these parameters, and on thresholds for these parameters [22-26]. We applied a similar approach to the question of an adherence rate threshold. Adherence rate thresholds have been previously defined in terms of the serum concentration of a drug, either for clusters of dosing patterns [25] or for individual patients [26]. The current study defined the adherence rate threshold based on the physiological effects of the drug (i.e., LDL-C concentration). Thus, we have extended the concept of PK adherence to include PD effects and now define pharmacometric adherence as any pattern of dosing that achieves or maintains therapeutic efficacy. 
Fig. 3 Predicted levels of LDL$\mathrm{C}$ at various adherence rates for a simvastatin $20 \mathrm{mg}$ and b atorvastatin $5 \mathrm{mg}$. The black dashed line shows the LDL-C goal of $<70 \mathrm{mg} / \mathrm{dL}$. $L D L$ $C$ low-density lipoprotein cholesterol

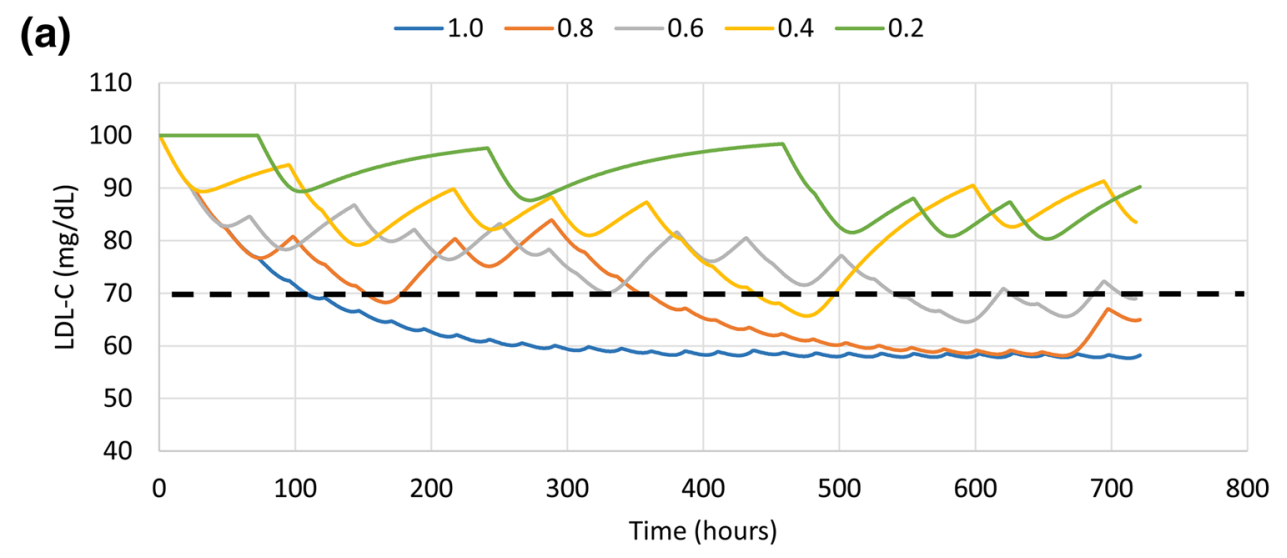

(b)
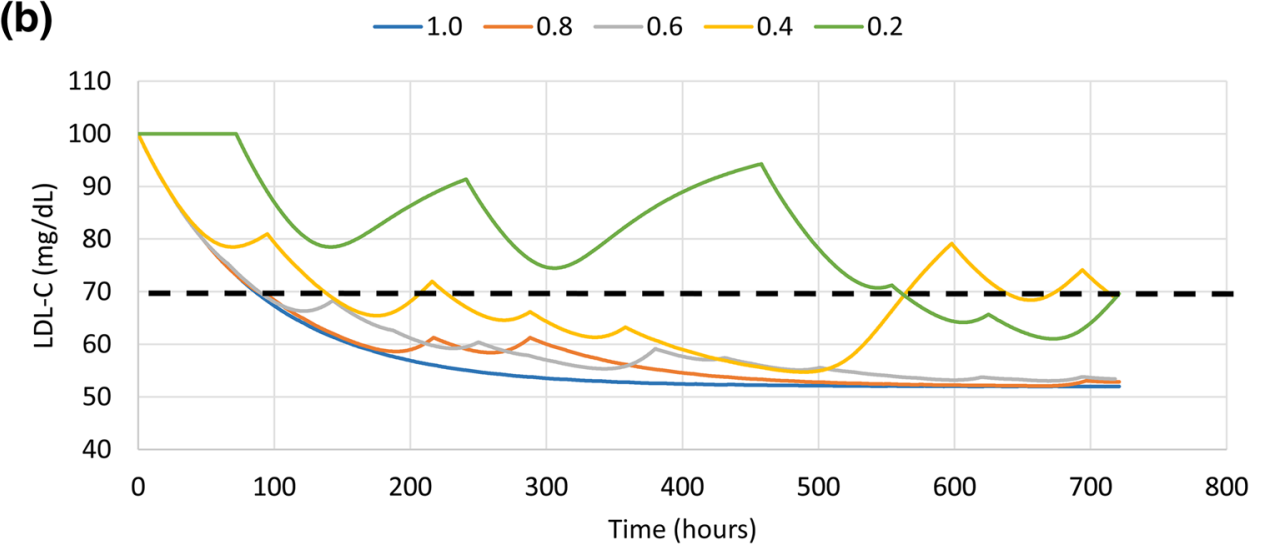

(a) 100

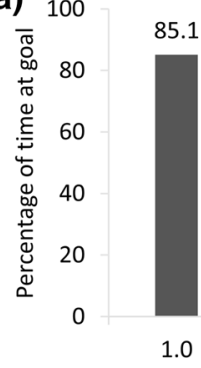

(b)

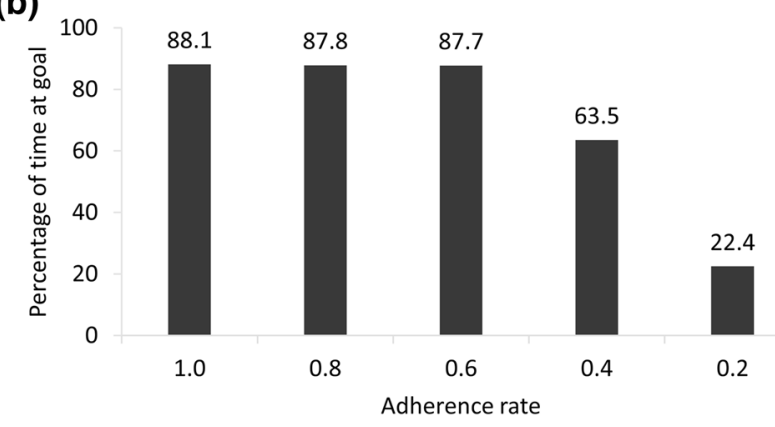

Fig. 4 Time at the $<70 \mathrm{mg} / \mathrm{dL}$ LDL-C goal with a simvastatin $20 \mathrm{mg}$ and b atorvastatin $5 \mathrm{mg}$. $L D L-C$ low-density lipoprotein cholesterol
Our findings are consistent with what is known about the PK properties of statins. Other variables being equal, drugs with longer half-lives and with indirect physiological effects are known to be more forgiving, i.e., less sensitive to variations in dosing [27]. All statins are somewhat forgiving, in part because of the delay between inhibition of HMG-CoA reduction and reduction of serum LDL-C levels. However, in addition to this feature, the plasma elimination half-lives vary widely among statins, with values of $2-5 \mathrm{~h}$ for simvastatin and $14 \mathrm{~h}$ for atorvastatin $[13,28]$. Thus, we hypothesized a priori that LDL-C would be less sensitive to missed doses of atorvastatin than to the same pattern of misses with simvastatin.

Our results are also consistent with the demonstrated success of alternate-day dosing with statins (which is a specific case of a 0.5 adherence rate) [29]. Most clinical trials of atorvastatin have shown that reductions in LDL-C are the same or only slightly less with alternate-day dosing than with daily dosing [30-33]. In a PD modeling simulation of the relationship between adherence to atorvastatin and serum levels of LDL-C, the calculated mean percentage reduction in LDL-C among patients with perfect adherence to atorvastatin $40 \mathrm{mg}$ over 6 months was 52.0 
versus $39.9 \%$ with alternate-day dosing [34]. Together with these findings, our results suggest that some statins can be administered less frequently than once daily, with no detrimental effects on LDL-C levels. Of course, LDL-C levels are an intermediate endpoint of statin treatment, the ultimate goal of which is to reduce the risk of cardiovascular events. Future modeling studies of the adherence rate threshold could potentially assess the effects of variable adherence on this risk.

For doctors and their patients, the adherence rate threshold can inform the choice of drug within a class, based on previous or anticipated adherence patterns. The dosage of the selected drug can also be adjusted to account for adherence patterns (i.e., a lower dose for patients with better adherence, as long as the desired clinical effect is being achieved). Steiner's seminal description of methods of assessing refill compliance introduced the concept of the "compliance-adjusted daily dose" [2]. More recently, an individualized method of drug selection and dose adjustment based on adherence patterns has been described for the oral anticoagulant agents warfarin and acenocoumarol [35]. Such individualized prescription patterns have the potential to reduce costs and prevent adverse effects over a long-term course of treatment.

One of the limitations of this study was the selection of five individual patients in the iAdherence database with specific adherence rates. The dosing patterns of these patients are not necessarily representative of all patients at any given adherence rate. Indeed, there are many possible dosing patterns corresponding to each adherence rate. The average of all of these combinations is a hypothetical dosing pattern in which the missed doses are evenly spaced over the dosing period. As a sensitivity analysis, we modeled LDL-C reduction for adherence rates of 0.8, 0.6, 0.4 , and 0.2 using hypothetical dosing patterns in which the missed doses were evenly spaced. The adherence rate thresholds for the sensitivity analysis were $>0.8$ for simvastatin and between 0.2 and 0.4 for atorvastatin (data not shown). The precision of these threshold values could be increased by incorporating a larger number of adherence rates (e.g., 0.1, 0.2, 0.3, 0.4, 0.5, etc.). However, the five adherence rates assessed in the current study were sufficient to illustrate the drug specificity of the adherence rate threshold.

Although only two drugs from a single class were assessed in this study, the principle can reasonably be expected to apply to any class of drugs whose members exhibit variations in their PK properties. As discussed in our previous publication, the forgiveness of a drug is dependent on its PK/PD properties, which are unique to that drug and which give rise to a unique adherence rate threshold [9].
The reductions in LDL-C observed with perfect adherence to simvastatin and atorvastatin were larger than the expected $30 \%$, based on a meta-analysis of clinical trial data [12]. However, the $42 \%$ reduction in LDL-C observed with perfect adherence to simvastatin $20 \mathrm{mg}$ is similar to the $\sim 38 \%$ reduction reported by Kim et al. for simvastatin $20 \mathrm{mg}$ after 6 weeks [13]. In addition, the PK/PD model was derived from Korean patient data [13], and Asian patients have been suggested in several studies to exhibit a heightened response to statin therapy [36-38].

\section{Conclusion}

In conclusion, this study showed that the adherence rate threshold is drug specific, suggesting that a universal threshold of 0.8 is inaccurate and inappropriate. It also illustrates the usefulness of PK/PD modeling for determining the adherence threshold for individual drugs and individual patients.

\section{Compliance with Ethical Standards}

Conflict of interest Melissa Stauffer, Paul Hutson, Anna Kaufman, and Alan Morrison have no competing interests to declare. Melissa Stauffer, Anna Kaufman, and Alan Morrison are affiliated with ScribCo, which received no external funding for this research. Paul Hutson has no financial relationship with ScribCo.

Open Access This article is distributed under the terms of the Creative Commons Attribution-NonCommercial 4.0 International License (http://creativecommons.org/licenses/by-nc/4.0/), which permits any noncommercial use, distribution, and reproduction in any medium, provided you give appropriate credit to the original author(s) and the source, provide a link to the Creative Commons license, and indicate if changes were made.

\section{References}

1. Cramer JA, Roy A, Burrell A, Fairchild CJ, Fuldeore MJ, Ollendorf DA, Wong PK. Medication compliance and persistence: terminology and definitions. Value Health. 2008;11:44-7. doi:10.1111/j.1524-4733.2007.00213.x.

2. Steiner JF, Prochazka AV. The assessment of refill compliance using pharmacy records: methods, validity, and applications. J Clin Epidemiol. 1997;50:105-16.

3. Andrade SE, Kahler KH, Frech F, Chan KA. Methods for evaluation of medication adherence and persistence using automated databases. Pharmacoepidemiol Drug Saf. 2006;15:565-74. doi:10.1002/pds.1230 (discussion 575-67).

4. Siris ES, Harris ST, Rosen CJ, Barr CE, Arvesen JN, Abbott TA, Silverman S. Adherence to bisphosphonate therapy and fracture rates in osteoporotic women: relationship to vertebral and nonvertebral fractures from 2 US claims databases. Mayo Clin Proc. 2006;81:1013-22. doi:10.4065/81.8.1013.

5. Caro JJ, Ishak KJ, Huybrechts KF, Raggio G, Naujoks C. The impact of compliance with osteoporosis therapy on fracture rates in actual practice. Osteoporos Int. 2004;15:1003-8. doi:10.1007/ s00198-004-1652-z. 
6. Bezabhe WM, Chalmers L, Bereznicki LR, Peterson GM. Adherence to antiretroviral therapy and virologic failure: a metaanalysis. Medicine (Baltimore). 2016;95:e3361. doi:10.1097/MD. 0000000000003361.

7. Viswanathan S, Justice AC, Alexander GC, Brown TT, Gandhi NR, McNicholl IR, Rimland D, Rodriguez-Barradas MC, Jacobson LP. Adherence and HIV RNA suppression in the current era of highly active antiretroviral therapy. J Acquir Immune Defic Syndr. 2015;69:493-8. doi:10.1097/QAI. 0000000000000643.

8. Watanabe JH, Bounthavong M, Chen T. Revisiting the medication possession ratio threshold for adherence in lipid management. Curr Med Res Opin. 2013;29:175-80. doi:10.1185/ 03007995.2013.766164.

9. Morrison A, Stauffer ME, Kaufman AS. Relationship between adherence rate threshold and drug 'forgiveness'. Clin Pharmacokinet. 2017; . doi:10.1007/s40262-017-0552-2 (in press).

10. Vrijens B, Vincze G, Kristanto P, Urquhart J, Burnier M. Adherence to prescribed antihypertensive drug treatments: longitudinal study of electronically compiled dosing histories. BMJ. 2008;336:1114-7. doi:10.1136/bmj.39553.670231.25.

11. D'Argenio D, Schumitzky A, Wang X. ADAPT 5 user's guide: pharmacokinetic/pharmacodynamic systems analysis software. Los Angeles: Biomedical Simulations Resource; 2009.

12. Law MR, Wald NJ, Rudnicka AR. Quantifying effect of statins on low density lipoprotein cholesterol, ischaemic heart disease, and stroke: systematic review and meta-analysis. BMJ. 2003;326:1423. doi:10.1136/bmj.326.7404.1423.

13. Kim J, Ahn BJ, Chae HS, Han S, Doh K, Choi J, Jun YK, Lee YW, Yim DS. A population pharmacokinetic-pharmacodynamic model for simvastatin that predicts low-density lipoprotein-cholesterol reduction in patients with primary hyperlipidaemia. Basic Clin Pharmacol Toxicol. 2011;109:156-63. doi:10.1111/j. 1742-7843.2011.00700.x.

14. Narwal R, Akhlaghi F, Asberg A, Hermann M, Rosenbaum SE. Development of a population pharmacokinetic model for atorvastatin acid and its lactone metabolite. Clin Pharmacokinet. 2010;49:693-702. doi:10.2165/11535980-000000000-00000.

15. Kakara M, Nomura H, Fukae M, Gotanda K, Hirota T, Matsubayashi S, Shimomura H, Hirakawa M, Ieiri I. Population pharmacodynamic analysis of LDL-cholesterol lowering effects by statins and co-medications based on electronic medical records. Br J Clin Pharmacol. 2014;78:824-35. doi:10.1111/bcp.12405.

16. Aoyama T, Omori T, Watabe S, Shioya A, Ueno T, Fukuda N, Matsumoto Y. Pharmacokinetic/pharmacodynamic modeling and simulation of rosuvastatin using an extension of the indirect response model by incorporating a circadian rhythm. Biol Pharm Bull. 2010;33:1082-7.

17. Dansette PM, Jaoen M, Pons C. HMG-CoA reductase activity in human liver microsomes: comparative inhibition by statins. Exp Toxicol Pathol. 2000;52:145-8. doi:10.1016/s09402993(00)80107-4.

18. Catapano AL, Graham I, De Backer G, Wiklund O, Chapman MJ, Drexel H, Hoes AW, Jennings CS, Landmesser U, Pedersen TR, Reiner Z, Riccardi G, Taskinen MR, Tokgozoglu L, Verschuren WM, Vlachopoulos C, Wood DA, Zamorano JL, Authors/Task Force M, Additional C. 2016 ESC/EAS guidelines for the management of dyslipidaemias. Eur Heart J. 2016;37:2999-3058. doi:10.1093/eurheartj/ehw272.

19. Stone NJ, Robinson JG, Lichtenstein AH, Bairey Merz CN, Blum CB, Eckel RH, Goldberg AC, Gordon D, Levy D, Lloyd-Jones DM, McBride P, Schwartz JS, Shero ST, Smith SC Jr, Watson K, Wilson PW, American Collegeof Cardiology/American Heart Association Task Force on Practice G. 2013 ACC/AHA guideline on the treatment of blood cholesterol to reduce atherosclerotic cardiovascular risk in adults: a report of the American College of
Cardiology/American Heart Association Task Force on Practice Guidelines. J Am Coll Cardiol. 2014;63:2889-934. doi:10.1016/j. jacc.2013.11.002.

20. Gieschke R, Steimer JL. Pharmacometrics: modelling and simulation tools to improve decision making in clinical drug development. Eur J Drug Metab Pharmacokinet. 2000;25:49-58.

21. Smith DA, Jones B. Variability in drug response as a factor in drug design. Curr Opin Drug Discov Dev. 1999;2:33-41.

22. Tu W, Morris AB, Li J, Wu J, Young J, Brater DC, Murray MD. Association between adherence measurements of metoprolol and health care utilization in older patients with heart failure. Clin Pharmacol Ther. 2005;77:189-201. doi:10.1016/j.clpt.2004.10. 004.

23. Dickinson L, Boffito M, Khoo SH, Schutz M, Aarons LJ, Pozniak AL, Back DJ. Pharmacokinetic analysis to assess forgiveness of boosted saquinavir regimens for missed or late dosing. J Antimicrob Chemother. 2008;62:161-7. doi:10.1093/jac/dkn187.

24. Schwalbe O, Scheerans C, Freiberg I, Schmidt-Pokrzywniak A, Stang A, Kloft C. Compliance assessment of ambulatory Alzheimer patients to aid therapeutic decisions by healthcare professionals. BMC Health Serv Res. 2010;10:232. doi:10.1186/ 1472-6963-10-232.

25. Maclean R, Pfister M, Zhou Z, Roy A, Tuomari VA, Heifets M. Quantifying the impact of nonadherence patterns on exposure to oral immunosuppressants. Ther Clin Risk Manag. 2011;2011:149-56.

26. Morrison A, Stauffer ME, Kaufman AS. Defining medication adherence in individual patients. Patient Prefer Adherence. 2015;9:893-7. doi:10.2147/PPA.S86249.

27. Boissel JP, Nony P. Using pharmacokinetic-pharmacodynamic relationships to predict the effect of poor compliance. Clin Pharmacokinet. 2002;41:1-6. doi:10.2165/00003088-20024101000001.

28. Schachter M. Chemical, pharmacokinetic and pharmacodynamic properties of statins: an update. Fundam Clin Pharmacol. 2005;19:117-25. doi:10.1111/j.1472-8206.2004.00299.x.

29. Marcus FI, Baumgarten AJ, Fritz WL, Nolan PE Jr. Alternate-day dosing with statins. Am J Med. 2013;126:99-104. doi:10.1016/j. amjmed.2012.08.007.

30. Ghia CJ, Panda AS, Khobragade LR, Jha RK, Rambhad GS. Alternate day versus once daily atorvastatin for primary prevention of (CHD) in naive patients of dyslipidemia. J Clin Diagn Res. 2014;8:27-31. doi:10.7860/jcdr/2014/7359.4096.

31. Jafari M, Ebrahimi R, Ahmadi-Kashani M, Balian H, Bashir M. Efficacy of alternate-day dosing versus daily dosing of atorvastatin. J Cardiovasc Pharmacol Ther. 2003;8:123-6.

32. Matalka MS, Ravnan MC, Deedwania PC. Is alternate daily dose of atorvastatin effective in treating patients with hyperlipidemia? The alternate day versus daily dosing of atorvastatin study (ADDAS). Am Heart J. 2002;144:674-7.

33. Pramanik S, Das AK, Chakrabarty M, Bandyopadhyay SK, Ghosh M, Dalai CK. Efficacy of alternate-day versus everyday dosing of atorvastatin. Indian J Pharmacol. 2012;44:362-5. doi:10.4103/0253-7613.96326.

34. Hughes DA, Walley T. Predicting "real world" effectiveness by integrating adherence with pharmacodynamic modeling. Clin Pharmacol Ther. 2003;74:1-8. doi:10.1016/S00099236(03)00091-2.

35. Blesius A, Chabaud S, Cucherat M, Mismetti P, Boissel JP, Nony $P$. Compliance-guided therapy: a new insight into the potential role of clinical pharmacologists. Clin Pharmacokinet. 2006;45:95-104. doi:10.2165/00003088-200645010-00007.

36. Itoh $\mathrm{T}$, Matsumoto M, Hougaku H, Handa N, Tsubakihara $\mathrm{Y}$, Yamada Y, Imaizumi M, Hoshi M, Shimazu Y, Hori M, Kawamori R, Ueda N, Fusamoto H, Kamada T. Effects of low-dose simvastatin therapy on serum lipid levels in patients with 
moderate hypercholesterolemia: a 12-month study. The Simvastatin Study Group. Clin Ther. 1997;19:487-97.

37. Lee E, Ryan S, Birmingham B, Zalikowski J, March R, Ambrose H, Moore R, Lee C, Chen Y, Schneck D. Rosuvastatin pharmacokinetics and pharmacogenetics in white and Asian subjects residing in the same environment. Clin Pharmacol Ther. 2005;78:330-41. doi:10.1016/j.clpt.2005.06.013.
38. Wu CC, Sy R, Tanphaichitr V, Hin AT, Suyono S, Lee YT. Comparing the efficacy and safety of atorvastatin and simvastatin in Asians with elevated low-density lipoprotein-cholesterol-a multinational, multicenter, double-blind study. J Formos Med Assoc. 2002;101:478-87. 